\title{
XXVIII. Mineralogische Mittheilungen ${ }^{1}$ ).
}

\author{
Von
}

F. Zambonini in Rom.

(Hierzu Taf. VIII, Fig. 7-11.)

\section{Natrolith rom Langesundfjord.}

(Hierzu Taf. VIII, Fig. 7-10.)

Die ersten genauen Messungen an den schönen Natrolithkrystallen dieses berühmten Fundortes wurden von G. Kose ${ }^{2}$, G. A. Kenngott ${ }^{3}$ ) und V. v. Lang ${ }^{4}$ ausgeführt. Aber wir verdanken den Untersuchungen W. (. Brögger's ${ }^{5}$ ) eine vollstïndige Monographie dieses Minerals, welches er in zwei wichtigen $\Lambda$ rbeiten sehr genau bekannt gemacht hat.

Ich habe vor Kurzem Gelegenheit gehalot, Stufen dieser Localität zu sludiren, und ich habe einige Krystalle gefunden, welche mir einige neue Formen geliefert haben. Iiese Krystalle bieten ein besonderes Interesse, weil oft ilıre Flächen eine ganz asymmetrische Entwickelung und alle von mir beobachteten Formen mit Ausnahme des Prisma $\{1.10\} \infty P$, der beiden verticalen Pinakoide und der Pyramide $\{111\} P$, im Allgemeinen nur eine einzige Fläche zeigen. Auch die neuen von mir gefundenen Formen kommen bloss mit einer Flïche vor, welche jedoch immer so gross, eben und gläuzend ist, diss sie genaue Messungen liefern und ilıre Symbole ganz sicher sind. Wichtig ist, dass dieselbe 'Thatsache auch an anderen Natrolithen beschrieben wurde. Aus dem bemerkenswerthen Aufsatz

1) Fortsetzung der min. Mitth. diese Zeitschr. 34, 225-260.

2) Das krystallochemische Mineralsystem. Leipzig 1852, S. 94.

3) Lebersicht der Resultate mineralogiseher Forschungen im Jahre 1852, S. $\$ 4$.

4) Maskelyne and Lang, Mineralogical Notes. Philos. Magazine, Jan. 1863.

5) Untersuchungen norwegischer Mincralien. Diese Zeitschr. 1879, 3, 478.

Die Mineralien der Syenitpegmatitgänge der süd-norwegischen Augit- und Nephelinsyenite. Diese Zcitschr. 1890, 16, 398. 
G. B. Negri's 1$)$ über den venetianischen Natrolith ergiebt sich, dass die Formen $\{434\} \bar{P}_{3}^{4},\{951\} 9 \bar{P} \frac{9}{5},\{331\} 3 P,\{511\} 5 \bar{P} 5,\{252\} \frac{5}{2} \breve{P}_{\frac{5}{2}},\{120\} \infty \breve{P}_{2}$ am Natrolith von Salcedo, $\{211\} 2 \bar{P} 2,\{772\} \frac{7}{2} P,\{885\} \frac{8}{5} P$ an Krystallen von Monte Baldo nur eine Fläche besitzen.

Fast immer sind die Flächen der zwei vorherrschenden Formen $\{110\}$ und $\{111\}$ ungleich entwickelt, und manchmal treten die Flächen von $\{111\}$ nur theilweise auf. Ein solcher Krystall ist in Fig. 7 Taf. VIIl dargestellt; an dem positiven Ende der $\Lambda$ xe $c$ laufen nur drei der vier oberen Flächen von \{111\} zusammen. Nach dieser Ausbildung ähneln meine Krystalle jenen vom Limberger Kopf, an welchen E. Kaise $\mathbf{r}^{2}$ ) beobachtet hat, dass die Pyramidenflächen vielfach ungleich ausgebildet sind und oft zwei, oft auch drei vollkommen zurücktreten.

Vicinalflächen kommen an meinen Krystallen nicht häufig vor, dennoch habe ich einige der schon bekannten Formen mit complicirten Symbolen

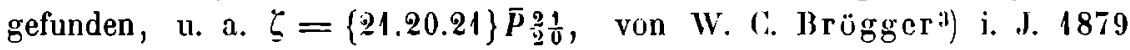
bestimmt; sie wurde auch von E. $\left.\Lambda \mathrm{rtini}^{4}\right)$ an Krystallen aus Bombiana, von $F^{2}$. (Gonnard ${ }^{5}$ ) an jenen aus der Auvergne beobachtet.

In diesem Aufsatze beschränke ich mich darauf, die Krystalle zu beschreiben, welche neue Formen geliefert haben, weil cine vollstindige Arbeit über meine: Krystalle nach den wichtigen $\Lambda$ bhandlungen C. W. Brögger's überflüssig wäre.

Die neuen, von mir bestimmten Formen sind folgende:

\begin{tabular}{|c|c|c|c|c|}
\hline Miller: & Na uman & Lévy: & Weiss: & Buchstabe: \\
\hline $\begin{array}{l}\{334\} \\
\{335\}\end{array}$ & $\begin{array}{l}3_{4}^{3} P \\
3 \\
3\end{array}$ & $\begin{array}{l}b^{\frac{2}{3}} \\
b_{6}^{5}\end{array}$ & $\begin{array}{ll}a: b: & \frac{3}{4} c \\
a: b: & \frac{3}{5} c\end{array}$ & $\begin{array}{l}g \\
k\end{array}$ \\
\hline$\{112\}$ & $\frac{1}{2} P$ & $b^{1}$ & $a: b: \frac{1}{2} c$ & $j$ \\
\hline$\{361\}$ & $6 \breve{P} 2$ & $b^{\frac{1}{3}} b^{\frac{1}{5}} g^{1}$ & $2 a: b: 6 c$ & $p$ \\
\hline$\{836\}$ & $\frac{4}{3} \bar{P}_{3}^{8}$ & $b^{\frac{1}{3}} b \mathrm{r}^{\frac{1}{1}} h^{\frac{1}{d}}$ & $\frac{3}{8} a: b: \quad \frac{1}{2} c$ & $\varepsilon$ \\
\hline$\{16.16 .17$ & \}) $\frac{1}{1} 7^{\circ} \mathrm{P}$ & $b^{13}$ & $a: b: \frac{16}{17} c$ & $\xi$ \\
\hline
\end{tabular}

Alle diese Formen habe ich an Krystallen beobachtet, welche vielleicht dem Typus II Brügger's zuzuschreiben sind. Nach Brügger, welcher die Natrolithkrystalle vom Langesundfjord in drei Typen theilte, gehüren dem zweiten, welcher ohne Zweifel der häufigsle ist, jene Krystalle an, "welche ..... nach der Verticalaxe prismatisch, durch flächenürmere Combinationen,

1) Note cristallografiche su di alcune natroliti venete e appunti crilici. Rivista di Mineralogia Ital. 1890, 7, 69. Ausz. dicse Zeitschr. 20, 628.

2) Natrolith vom Límberger Kopf bei Asbach u. s. w. Diese Zeitschr. 1899, 31, 32.

3) a. a. 0 .

4) Sulla Natrolite di Bombiana nel Bolognese. Pendiconti R. Accad. Lincei, Classe- scienze fis. mat. e nat., 6. Jan. 1890 , Serie 4, 5, 37. Ref. diese Zeitschr. $20,172$.

5) Notes cristallographiques sur la mesotype du Puy-de-Dome. Bull. soc. franç. min. 1899, 15, 221. Ausz. diese Zeitschr. 24, 615. 
häufig nur $\{110\} \infty P$ und die beiden verticalen Pinakoide, sowie $\{111\} P$ und eine oder mehrere zu $P$ vicinalen Pyramiden, charakterisirt sind k. Meine Krystalle zeigen aber, wie ich schon gesagt habe, nur selten Vicinalflächen.

Um die Winkel der neuen Formen zu berechnen, habe ich die Constanten angewandt, welche W. C. B rügger schon 1879 für diesen Typus feststellte, nümlich:

$$
a: b: c=0,97856: 1: 0,353628 \text {. }
$$

Einige der von mir für die Formen $\{110\}$ und $\{111\}$ gemessenen Winkel sind in wenig befriedigender Uebereinstimmung mit den berechneten Werthen, aber die Unbeständigkeit der Winkel ist an Natrolith ein häufiges Phänomen. Besonders wichtig für diesen Punkt sind die Arbeiten von E. $\left.\Lambda \mathrm{rtini}^{1}\right)$ und G. B. $\mathrm{Negri}^{2}$ ) über den Natrolith aus dem Venetianischen, aus welchen folgt, dass die Krystalle von sehr nahestehenden Localitäten abweichende Constanten und jene desselben Fundortes starke Verschiedenheiten in den Winkeln zeigen. So variirt nach $\Lambda \mathrm{rtini}$ der Winkel (110) (III) an Krystallen von Montecchio Maggiore zwischen $62^{\circ} 55^{\prime}$ und $64^{0} 7^{\prime}$; an jenen von Monte Baldo zwischen 69045' und 64032'.

An demselben Krystalle fand ich dic neue Form $\varepsilon=\{836\}_{3}^{4} \bar{P}_{3}^{8}$ und die zu $\{111\} J^{\prime}$ vicinale Pyramide $\xi\{16.16 .17\} 1{ }_{17}^{16} P$. Die erstere zeigt eine grosse, elone und glïnzende Fläche, welche genaue Messungen lieferte. Zwischen (1T/) und (T11) findet sich genau in der Zone [1T0:1Ti] dic kleinere Iläche von $\{16.16 .17\}$. (iemessene Winkel:

$$
\begin{aligned}
(100):(836) & =64^{\circ} 45^{\prime} \text { gem. } \\
(010):(836) & =81 \quad 5037^{\prime} \text { ber. } \\
(111):(836) & =1047-8057- \\
(16 . \overline{1} 6.17):(110) & =6432-656-
\end{aligned}
$$

Dieser Krystall, welcher ferner die Formen $\{100\}\{010\}\{110\}\{111\}$ zejgl, ist in Fig. 8 Taf. VIII dargestellt. Dic Flïchen der zwei Pinakoide sind etwas unregelmïssig; (100) ist grösser als $(\bar{T} 00)$, und von $\{010\}$ ist nur eine Flïche anwesend. Die vier Flächen von $\{111\}$, welche vorhanden sind, haben sehr verschiedene Grösse, wie die Figur zeigt. Die Prismenfliichen finden sich nicht in gleicher Beschaffenheit; (110) ist eben und glänzend und besitzt nur einige dürftige krumme Streifen in seinem unteren Theile, auch recht regelmüssig ist (T10); im Gegensatze dazu sind (1T0) und

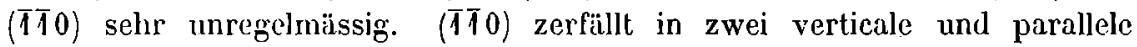
fliichenelemente, deren eines glïnzend und polyëdrisch, das andere parallel der $\Lambda$ xe $\approx$ stark gestreift ist und daher faserig erscheint.

1) Natrolite della Regione Veneta. Memorie R. Accad. Lincei, Classe scienze fis. mat. e nat. 1887, Seric 4, 4, 76. Ausz. diese Zeitschr. 14, 507.

2) a. a. 0 . 
Sehr merkwürdig ist der Krystall Fig. 9 Taf. VIII, welcher ausser den gewühnlichen Formen $\{100\}, 010\},\{110\},\{111\}$ auch die Basis $\{001\}$ mit einer ziemlich grossen und glänzenden Fläche und ferner die zwei neuen Protopyramiden $g=\{334\}\}_{4}^{3} P$ und $j=\{112\}_{2}^{1} P$ zeigt. Die Basis ist am Natrolith sehr selten; sie wurde von V. v. Lang und später von W. C. Brögger an Krystallen vom Lungesundfjord nachgewiesen. Die zwei neuen Pyramiden zeigen, wie die anderen schon erwähnten Formen, nur eine grosse Fläche. Die Fläche (3334) ist grösser als (1T2); letztere ist aber viel glïnzender. Beide haben sehr genaue Messungen geliefert:

$$
\begin{aligned}
& (3 \overline{3} 4):(1 \bar{T} 0)=69^{0} 22^{\prime} \text { gem. } \quad 69^{0} 14^{\prime} \text { ber. } \\
& (1 \bar{T} 2):(1 \bar{T} 0)=76 \quad 0 \quad-\quad 7549-
\end{aligned}
$$

Auch an diesem Krystalle zeigen die Flïchen von $\{111\}$ und $\{110\}$ sehr ungleiche Entwickelung. Wie die Figur zeigt, ist der Krystall etwas tafelrörmig nach dem Flïchenpaar (110) und (TT0).

In Fig. 10 Taf. VIII ist der Krystall dargestellt, an welchem ich die neuen Formen $p=\{361\} 6 \breve{P} 2$ und $k=\{335\} \frac{3}{5} P$ gefunden habe. $\{361\}$ bietet cine grosse und glïnzende Fläche; $\{335\}$ stumpft mit einer kleineren und sehr glänzenden Fläche die Kante $[\overline{T 11}: 1 \overline{1} 1]$ ab. Folgende Winkel wurden gemessen:

$$
\begin{aligned}
& (3 \overline{6} 1):(\overline{1} 0)=740 \quad 0^{\prime} \text { gem. } \\
& (3 \overline{6} 1):(1 \overline{1} 1)=4244^{\prime} \text { ber. } \\
& (3 \overline{6} 1):(0 \overline{1} 0)=3441-3217- \\
& (\overline{3} 35):(\overline{1} 11)=957-3448-
\end{aligned}
$$

\begin{tabular}{|c|c|c|c|}
\hline$a$ & $\{100\}$ & $\infty \bar{P} \infty$ & J. R. ILaüy \\
\hline$b$ & $\{010\}$ & $\infty \breve{P}_{\infty}$ & J. R. II a üy \\
\hline$c$ & $\{001\}$ & $0 P$ & V. v. Lang \\
\hline$m$ & $\{110\}$ & $\infty P$ & J. IR. II aüy \\
\hline$n$ & $\{120\}$ & $\infty \breve{P}_{2}$ & V. v. Lang \\
\hline$\lambda$ & $\{590\}$ & $\infty \breve{P}_{\bar{y}}$ & W. C. Brögger \\
\hline$\nu$ & $\{740\}$ & $\infty \bar{P}_{-\frac{7}{4}}$ & W. C. Brögger \\
\hline$t$ & $\{210\}$ & $\infty \bar{P} 2$ & E. $\operatorname{Artini}$ \\
\hline$l$ & $\{310\}$ & $\infty \bar{P} 3$ & W. C. Brögger \\
\hline$s$ & $\{011\}$ & $\breve{P} \infty$ & G. $\Lambda$. Kenngott \\
\hline$h$ & $\{031\}$ & $3 \breve{P} \infty$ & V. v. Lang \\
\hline$D$ & $\{101\}$ & $\overline{\boldsymbol{P}} \infty$ & E. Artini \\
\hline
\end{tabular}

Wie an den vorigen Krystallen, sind die Flächen von $\{111\}$ und $\{110\}$ unsymmetrisch entwickelt: die zwei liächen $(1 T 0)$ und $(\pi / 0)$ sind vorherrschend.

Mit den von mir beobachteten steigt die Zahl der bis jetzt am Nalrolith beslimmten Formen auf 4h, nïmlich: 


\begin{tabular}{|c|c|c|c|}
\hline$u$ & $\{301\}$ & $3 \overline{\boldsymbol{P}} \infty$ & V. v. Lang \\
\hline$v$ & $\{601\}$ & $6 \bar{F} \infty$ & A. Seligmann \\
\hline 0 & $\{111\}$ & $P$ & J. R. Haüy \\
\hline$d$ & $\{221\}$ & $2 P$ & A. Dufrénoy \\
\hline$\approx$ & $\{331\}$ & $3 P$ & V. v. Lang \\
\hline$g$ & $\{331\}$ & $\frac{3}{4} P$ & F. Zambonini \\
\hline$k$ & $\{335\}$ & $\frac{3}{5} I^{\prime}$ & F. Zambonini \\
\hline$\omega$ & $\{551\}$ & $5 P$ & W. C. Brügger \\
\hline$e$ & $\{772\}$ & $\pi$ & G. B. Negri \\
\hline$i$ & $\{885\}$ & $\frac{8}{5} T$ & G. B. Negri \\
\hline$j$ & $\{112\}$ & $\frac{1}{2} P$ & F. Zambonini \\
\hline$y$ & $\{131\}$ & $3 \breve{P} 3$ & G. Rose \\
\hline$w$ & $\{252\}$ & $\frac{5}{2} \breve{P}$. & G. B. Negri \\
\hline$\mu$ & $\{361\}$ & $6 \breve{P} 2$ & F. Zambonini \\
\hline r & $\{391\}$ & $9 \breve{P 3}$ & V. v. I ang \\
\hline$\beta$ & $\{434\}$ & $\bar{P}_{3}^{4}$ & G. B. Negri \\
\hline$\varepsilon$ & $\{836\}$ & $-\frac{4}{3} \overline{8}$ & F. Zambonini \\
\hline$\delta$ & $\{951\}$ & $\mathbf{9} \bar{P}$ & G. B. Negri \\
\hline$\gamma$ & $\{2 \mid 1\}$ & $2 \bar{P} \mathscr{2}$ & G. B. Negri \\
\hline$q$ & $\{3 \mid 1\}$ & $3 \bar{P} 3$ & $\Lambda$. Seligmann \\
\hline$\vartheta$ & $\{511\}$ & $\zeta \bar{P} 5$ & W. C. Brögger \\
\hline$\varrho$ & $\{12.12 .5\}$ & ${ }_{5}^{12} P$ & E. Palla \\
\hline$\xi$ & $\{16.16 .17\}$ & ${ }_{17}^{16} P$ & F. Zambonini \\
\hline$\sigma$ & $\{31.31 .30\}$ & $30 P$ & E. Palla \\
\hline 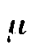 & $\{31.30 .0\}$ & $\infty \bar{P}_{31}^{31}$ & E. Palla \\
\hline$x$ & $\{11.10 .11\}$ & $\bar{P}: 1$ & l'hillips \\
\hline$\pi$ & $\{34.36 .1\}$ & $36 P_{17}^{18}$ & W. C. Brügger \\
\hline$\tau$ & $\{40.44 .43\}$ & ${ }_{4}^{4} \breve{p}_{10}^{1}$ & E. Palla \\
\hline$\eta$ & $\{21.19 .21\}$ & $\bar{P} \frac{21}{19}$ & Lévy \\
\hline$\zeta$ & $\{21.20 .21\}$ & $\bar{P}_{20}^{21}$ & W. C. Brügger \\
\hline$\varphi$ & $\{27.25 .27\}$ & $\left.\bar{P}_{2}^{2}\right\}$ & E. Palla \\
\hline$Q$ & $\{40.40 .39\}$ & $40 P^{2}$ & F. Gonnard. \\
\hline
\end{tabular}

Die Formen $\omega=\{551\} 5 P$ und $r=\{511\} 5 \bar{P} 5$ wurden fast gleichzeitig von W. C. Brögger ${ }^{1}$ ) und E. Artini $\left.{ }^{2}\right)$ an Krystallen vom Langesundfjord resp. Monte Baldo gefunden. Dic Abhandlung Brögger's ist aber einige Monate früher als die Arbeit von Artini erschienen. Die Formen, welche zuerst $\Lambda$. Seligmann ${ }^{3}$ ) bestimmte, kamen an Krystallen von Salesl

1) Geolog. För. i Stockholm Förhandlingar 1887, 9, 266.

2) Sopra alcuni nuovi cristalli interessanti di Natrolite del Monte Baldo. Rendiconti R. Accad. dei Lincei, Classe di scienze fis., mat. e nat. 1887, 3, 2. Semestre, 215. Ausz. diese Zeitschr. 14, 318.

3) Mineralogische Notizen. Diese Zeitschr. 1878, 1, 398. 
vor, wie die Vicinalen, welche E. Palla 1 ) entdeckte. In vorstehendem Verzeichnisse ist die Form $\{20.20 .1\} 20 P$ weggelassen, welche nach E. Artini ${ }^{2}$ ) sehr zweifelhaft ist und undeutliche Kanten mit $\{110\}$ bildet.

W. G. Brügge r hatte in scinen Arbeiten für das Prisma $l=\{310\} \infty \bar{P} 3$ das Symbol $\{610\} \infty \bar{P} 6$ berechnet, aber aus dem von ihm gemessenen Winkel

$$
l: a=173^{\prime}
$$

folgt für $l$ das Symbol $\{310\}$.

Die Prismenflächen zerfallen häufig in viele Flächenelemente, welche unter einander sehr wenig geneigt sind. Manchmal ist die Polyëdrie ungleich entwickelt an den verschiedenen Flächen des Prismas;' es giebt Krystalle, an denen nur eine Fläche des Prismas Polyëdrie zeigt, bisweilen sind aber zwei oder alle polyëdrisch.

\section{Pyrosmalith ans der Bjelke-Grube (Nordmarken).}

Der Pyrosmalith wurde von II. Gahn und Clason entdeckt und unter den Namen »'Pyrodmalith * von J. F. L. Hausmann') beschrieben. llausmann beobachtete nur sechsseitige Prismen, bald als Süule, bald als Tafeln. In demselben Jahre 1808 wurde der Name in die heutige Form "Pyrosmalith * von Karsten ) umge:̈ndert.

IR. J. Ila ü $y^{5}$ ) hat unter dem Namen $•$ Fer muriatéa den Pyrosmalith als monoklin beschrieben, und F. S. Beudant ${ }^{6}$ ) betrachtete ihn als eine Diopsidvarietät. Diese ungenauen Beobachtungen wurden aber schon im Jahre 1821 von $R$. Jameson ${ }^{7}$ ) und später von $A$. Breithaupt $\left.{ }^{8}\right)$ berichtigt. IJem Letzteren verdanken wir sehr wichtige Notizen über die krystallographischen und physikalischen Eigenschaften des Pyrosmalith; er machte auf die Einaxigkeit des Minerals aufmerksam und führte neue Bestimmungen des specifischen Gewichts aus. Auch F. Mohs") und A. Dufrénoy ${ }^{10}$ ) hielten den Pyrosmalith für hexagonal, und Letzterer hat auch die $\Lambda$ nnabme von IIisinger, Berzelius und Beudant widerlegt, nach welcher im Pyrosmalith das Eisenchlorid zufïllig beigemischt wïre.

1) Leber die vicinalen Pyramidenflächen ann Natrolith. Diese Zeitschr. 1884, 9, 386.

2) a. a. 0 .

3) Moll's Ephemeriden der Berg- und Ifüttenkunde 4\$08, 4, 390.

4) Mineralogische Tabellen, Berlin 1808, S. 103.

5) Traité de Minéralogie, qième Édition, Paris 1822, 4, 138.

6) Traité élémentaire de Minéralogie, Paris $1830,2,228$.

7) Manual of Mineralogy, Edinburgh 1821, S. 329. Auch: $\Lambda$ System of Mineralogy, 36. Edition, 3, 361 .

8) Vollstiandige Charakteristik des Mineralsystems, Dresden und Lejpzig 1832, 93.

9) Leichtfassliche Anfangsgründe der Naturgeschichte des Mineralreiches. Zweite Aullage, Wien $1839,2,226$.

10) Traité de Minćralogie, Paris $1845,2,549$. 
Der Frste jedoch, welcher die Krystallformen des Pyrosmaliths genau beschrieben hat, ist II. J. Brooke $\left.{ }^{1}\right)$. Die von ihm beobachteten Formen sind das hexagonale Prisma $\{10 \bar{T} 0\} \infty P$, die Basis $\{0001\} 0 P$ und zwei Pyramiden, nämlich $\{10 \overline{1} 1\} P^{\prime}$ und $\{20 \overline{2} 1\} 2 P$. Seine Constanten, aus dem Winkel

$$
\left.(10 \overline{1} 1):(0001)=129013^{\prime 2}\right)
$$

berechnet, wurden auch von A. Des Cloizeaux ${ }^{3}$ ) angenommen. Sie sind jenen, welche $\Lambda$. E. Nordenskiöld ${ }^{4}$ ) viele Jahre spüter berechnete, niimlich:

sehr nahe.

$$
e=0,5308
$$

Die Meinung II äy's und Beudant's, dass der Pyrosmalith nicht hexagonal ist, wurde von $N$. v. Nordenskiöld5) später wieder anfgenommen. Er grründete sie auf dic Streifen, welche sich manchmal auf der Basis der Pyrosmalithkrystalle linden, genau wie N. v. Kokscharow ${ }^{6}$ ) aus demselben Grunde den Molybdinit als rhombisch oder monoklin betrachtet hatte. Alıer F, v. Kobell ${ }^{7}$ ) bewies mit sorgfältigen stauroskopischen Untersuchungen, dass der lyrosmalith ohne Zweifel einaxig isl, und dann sprach auch N. v. Kokscharow sich gegen die Annahme N. vo Nordenskiold's aus. Des (iloizeaux") hestitigte dic Untersuchungen lv. v. Kobell's und stellte fest, dass Pyrosmalith oplisch negativ sei.

Alle Krystalle, an welchen oben erwïhnte Beobachtungen ausgeführt wurden, stammen aus der Bjelkegrube, nahe Philipstad; sie wurden auch sehr kurz von M. II ürnes ${ }^{10}$ ) und D. J. Wiser ${ }^{11}$ ) erwähnt: beide beobachteten die einfache Combination $\{0001\}\{10 \bar{T} 0\}$.

Die Krystalle von Dannemoril wurden erst im Jahre 1888 von G. Holm ${ }^{12}$ ) beschrieben.

1) Philos. Magazine 1897, Nr. 37, S. 261.

2) Innerer Winkel.

3) Manuel de Minćralogie, Paris 1862, S. 520.

4) Öfversigt af k. Vetenskaps-Akad. Förh. 1870, 27, 562.

ij) Bulletin de la Classe physico-malhematique de l'Acad. Imp. des Sciences de St. Pétersbourg 14, 318

6) Materialien zur Mincralogic Russlands, St. Petersluurg $185 \mathrm{~h}, \mathbf{2}, 267$.

7) Gelehrte Anzeigen der k. bayer. Akad. der Wissensch., München 1855, 16. Juli, Nr. 7, S. 63 und 1856, 28. Juli, Nr. 4, S. 29.

8) Materialien zur Mineralogie Russlands, St. Petersburg 1857, 2, $3: 1$.

9) Manuel de Minéralogie, Paris 1862, 1, 5z0.

10) Mittheilungen über die Mineraliensammlung der Frau Johanna Edlen von Henickstein. N. Jahrb. f. Min., Geognosie, Geol. u. s. w. 1846, 775.

11) Briefliche Mittheilung in N. Jahrb. f. Min., Geogn., Geol. u. s. w. 186:, 727.

12) Om förekomsten af kristalliserad pyrosmalith vid Dannemora. Geol. Förening. i Stockholm Förhandl. 1888, 10, 18. Ref. diese Zeitschr. 17, 129. 
E. S. Dana 1) und P. Groth 2) haben nach den Untersuchungen E. Bertrand's ), welcher den rhomboëdrischen Charakter des mit dem Pyrosmalith isomorphen Friedelit feststellte, auch unser Mineral als rhomboëdrisch betrachtet, obwohl die Entwickelung der Pyramidenflächen genau hexagonal ist.

Was nun die chemischen Eigenschaften des Pyrosmalith anbetrifft, so ist jeder ihn untersuchende Chemiker zu einer eigenen Formel gelangt.

Die zwei ersten Analysen hat W. H isinger ${ }^{4}$ ) ausgeführt, aber sie sind beide sehr unvollkommen. Die pyrognostischen Reactionen des Pyrosmalith haben H. (Gahn und J. Berzelius ${ }^{5}$ ) bekannt gemacht.

Vollständige Bestimmungen hat zuerst J. Lang ${ }^{6}$ ) veröffentlicht. Aus seinen Untersuchungen folgt die Formel

$$
\mathrm{RCl}_{2} .12 \mathrm{RO}^{2} 11 \mathrm{SiO}_{2}+8 \mathrm{H}_{2} \mathrm{O} \text {. }
$$

Jie Analysen Lang's wurden auch von G. A. Kenngolt i) berechnet; er gab die Formel

$$
5 \dot{R}^{3} \dddot{S} \ddot{i}+\dot{H}^{3} \ddot{S},
$$

worin $\dot{l}=F e O$ und $M n O$ im Verhältniss von $3: 2$ und wobei in $\dot{h}$ der 15. Theil des Sauerstoffs durch Chlor vertreten ist.

$\Lambda$ uch F. Wöhler ${ }^{8}$ ) hat eine unvollendete Analyse geliefert, aus welcher F. C. Rammelsberg ${ }^{9}$ ) die Formel

$$
\mathrm{RCl}_{2}+7 \mathrm{RSiO}_{3}+5 \mathrm{H}_{2} \mathrm{O}
$$

berechnete. Die Analyse Wöhler's gab aber zuviel Chlor und sie ist als unrichtig zu betrachten.

Wir verdanken E. Ludwig ${ }^{10}$ ) eine Reihe chemischer Untersuchungen am Pyrosmalith. Nach ihm ist das Eisen nur als Ferrosalz anwesend, und ist die chemische Zusammensetzung unseres Minerals durch die Formel

darstellbar.

$$
\mathrm{RCL}_{2} .9 \mathrm{RO}, 8 \mathrm{SiO}_{2}+7 \mathrm{H}_{2} \mathrm{O}
$$

1) Systen of Mineralogy, London 1892, S. 465.

2) Tabellarische Uebersicht der Mineralien, Braunschweig 1898, S. 127.

3) Note sur la forme cristalline de la Friedelite, Paris 1876, 1 p., separ. erschienen. Dicse Zeitschr. 1877, 1, 86.

4) Magazin der Gesellschaft nat. Fr. zu Berlin, 6. Jahrg., \{th., S. 311. - Schweigger's Journat f. Chemie $1815, \mathbf{2 3}, 54$.

5) De l'emploi clu chalumeau etc., Paris 1821, S. 243 .

6) Ueber den Pyrosmalith. Journal f. prakt. Chemie 1861, 83, 42\%.

7) Cebersichl der Resultate mineralogischer Forschungen im Jahre 1861, Leipzig 1862 , S. 49.

8! Nachrichten der Götting. Gesellsch. d. Wissensch. 1870, Nr. 20, und Liebig's Annalen $1870,156,8 ;$.

9) Handbuch der Mineralchemie 1875, 1, 686.

10) Ueher den P'yrosmalith. Tschermak's mineralogische Mittheilungen 4875, $2,11$. 
Aber diese l'ormel ist nur dann in guter Uebercinstimmung mit der Analyse, wenn Kalk und T'alkerde vernachlässigt werden. Wonn wir jedoch, was richliger ist ${ }^{1}$, auf die gefundene Menge von $\mathrm{CaO}$ und $\mathrm{MgO}$ Gewicht legen, so wird die Analyse Isudwig's (I):

1.

\begin{tabular}{lr}
$\mathrm{SiO}_{2}$ & 33,95 \\
$\mathrm{FeO}$ & 49,36 \\
$\mathrm{H}_{2} \mathrm{O}$ & $\mathbf{8 , 1 4}$ \\
$\mathrm{Cl}$ & 4,78 \\
$\mathrm{He}$ & 3,77 \\
\cline { 2 - 2 } & $\mathbf{1 0 0 , 0 0}$
\end{tabular}

II.

$$
33,04
$$$$
49,56
$$$$
8,67
$$$$
4,88
$$$$
\frac{3,85}{100,00}
$$

$\delta$

0,91

0,20

0,53

0,10

$\frac{0,08}{1,82}$

Diese Analyse entspricht gut der Formel

$$
R C \eta_{2} \cdot 10 R O .8 \mathrm{SiO}_{2}+7 \mathrm{H}_{2} \mathrm{O},
$$

welche die unter II stehenden Zahlen erfordert.

Mit Ludwig's Formel ist dic Summe der Diff. $\delta=4,88$.

In neuerer Zeit haben N. Engström²) und A. Gorge ${ }^{3}$ ) die Varietät von Dannemora untersucht. Der Erstere berechnete die Formel

$$
\mathrm{RCl}_{2} \cdot 15 \mathrm{RO} .12 \mathrm{SiO}_{2}+8 \mathrm{H}_{2} \mathrm{O}
$$

aber mit der Analyse (I) ist in besserer Uebereinstimmung die Formel

\begin{tabular}{|c|c|c|c|}
\hline & I. & II. & $\delta$ \\
\hline $\mathrm{SiO}_{2}$ & 33,29 & 34,03 & $0,7 / k$ \\
\hline $\mathrm{FeO}$ & 52,16 & 51,99 & 0,17 \\
\hline $\mathrm{II}_{2} \mathrm{O}$ & 7,18 & 7,43 & 0,25 \\
\hline$C l$ & 3,44 & 3,66 & 0,22 \\
\hline$F^{\prime} C$ & 2,72 & 2,89 & 0,17 \\
\hline \multirow[t]{2}{*}{$\mathrm{Al}_{2} \mathrm{O}_{3}$} & 1,21 & - & - \\
\hline & 100,00 & $10 \overline{000}$ & 1,55 \\
\hline
\end{tabular}
welche erfordert II:

$$
\mathrm{RCl}_{2} .14 \mathrm{RO} .11 \mathrm{SiO}_{2}+8 \mathrm{II}_{2} \mathrm{O}
$$

Mit der Formel Engström's ist die Summe von $\delta=1,75$.

Nach Gorgeu enthält der Pyrosmalith von J)annemora 2,92\% $\mathrm{Fe}_{2} \mathrm{O}_{3}$; nur in seiner Analyse wurde bis jetzt Eisenoxyd nachgewiesen. Jie von ihm vorgeschlagene Formel ist:

$$
\mathrm{RCl}_{2} .4 \mathrm{RO} .11 \mathrm{SiO}_{2}+9 \mathrm{H}_{2} \mathrm{O}
$$

1) Siehe darüber: P. Groth a. a. 0 .

2) Analys af s. k. ljus knebelit från Dannemora. Geologiska För. i Stockholm Förlaandlingar 18i6, 3, 116.

3) Sur la friedelite et la pyrosmalite. Compl. rend. 1884, 98 $\mathbf{3 8 6}$. Ausz. diese Zeitschr. 11, 188. Die Analyse des Pyrosmalith wurde auch in Bullet. soc. min. de rrance $1884,7,38$ etwas verändert veröffentlicht. 
aber die Formel (siehe unter II)

$$
\mathrm{RCl}_{2} .12 \mathrm{RO} .10 \mathrm{SiO}_{2}+9 \mathrm{H}_{2} \mathrm{O}
$$

entspricht besser den gefundenen Zahlen (1):

I.

$\begin{array}{lr}\mathrm{SiO}_{2} & 34,00 \\ \mathrm{HeO} & \mathbf{4 8 , 0 2} \\ \mathrm{Fe}_{2} \mathrm{O}_{3} & 2,91 \\ \mathrm{H}_{2} \mathrm{O} & \mathbf{8 , 4 9} \\ \mathrm{Cl} & 3,67 \\ \mathrm{He} & 2,91 \\ & 100,00\end{array}$

II.

$$
\text { 34, } 22
$$$$
4.9,29
$$$$
\begin{array}{rr}
- & - \\
9,24 & 0,75 \\
4,05 & 0,38 \\
3,20 & 0,29 \\
100,00 & -2,91
\end{array}
$$

Nach Gorgeu wäre die Summe von $\delta=4,07$.

Aus den bis jetzt bekannten Analysen kann man also folgende Formeln berechnen:

$$
\begin{aligned}
& R \mathrm{Cl}_{2} .12 \mathrm{RO} .11 \mathrm{SiO}_{2}+8 \mathrm{H}_{2} \mathrm{O} \text { aus der Analyse Lang's } \\
& \mathrm{RCl}_{2} .14 \mathrm{RO} .1 \mathrm{\textrm {SiO } _ { 2 }}+8 \mathrm{H}_{2} \mathrm{O} \text { - } \quad \text { - Engström's } \\
& \mathrm{RCl}_{2} .12 \mathrm{RO} .10 \mathrm{SiO}_{2}+9 \mathrm{H}_{2} \mathrm{O}-\mathrm{O} \quad \text { - Gorgeu's } \\
& \mathrm{RCl}_{2} \text { 10 } 0 \mathrm{RO} .8 \mathrm{SiO}_{2}+7 \mathrm{H}_{2} \mathrm{O} \text { - - - Iudwig's. }
\end{aligned}
$$

In den verschiedenen Analysen finden sich $\mathrm{FeO}$ und $\mathrm{MnO}$ in folgendem Verhällnisse :

$$
\begin{aligned}
& \mathrm{FCO}: \mathrm{MnO} \\
& \text { 1,14: } 1 \text { Lang } \\
& \text { 1,37: I Wüh]cr } \\
& \text { 1,04: } 1 \text { Ludwig } 1 \\
& 1: 1,06 \text { Engstrüm } \\
& 1: 1,05 \text { Gorgeu. }
\end{aligned}
$$

Ich halse neue chemische und krystallographische Untersuchungen an Krystallen von Nordmarken ausgeführt, deren liesultat kurz mitgetheilt werden möge.

Die von mir untersuchten Krystalle zeigen gewöhnlich die einfache Combination $\{0001\}\{10 \bar{T} 0\}$; aber manchmal sind die Combinationskanten dieser zwei Formen durch ein kleines $\{h \hat{h} h \hat{h} l\}$ abgestumpft. Bisweilen ist diese Pyramide wenig glïnzend und dann giebt sie etwas unsichere Messungen; aber es giebt auch Krystalle, an welchen sie ziemlich gross und sehr glänzend vorkommt. $Z$ wei sehr genaue Messungen lieferten:

$$
(10 \bar{T} 0):(h 0 \bar{h} l)=50^{\circ} 42^{\prime} \text { und } 50^{\circ} 4.8^{\prime} \text {, Mittel } 50^{\circ} 45^{\prime} \text {. }
$$

Ein dritter ungenauer Werth ist $50^{\circ} 30^{\circ}$ ca.

Wenn wir für $c$ den von $A$. E. Nordenskiöld angenommenen Werth

$$
c=0,5308
$$


anwenden, so erhalten wir für $(h 0 \bar{h} l)=(30 \overline{3} 4)$ :

$$
(1070):(3034)=50^{\circ} 45^{\prime} \text { ber. }
$$

Jie Constanten Nordenskiöld's sind also in völliger Uebereinstimmung mit den von mir gemessenen Winkeln. Die hexagonale Pyramide $t=\{3034\}$ ${ }_{1}^{3} P$ ist für den l'yrosmalith neu.

Wenn wir mit E. S. I) ana und P. Groth den Pyrosmalith als rhomboëdrisch betrachten, so sind alle ljis jetzt an diesem Mineral bekannten Formen folgende:

\begin{tabular}{|c|c|c|c|c|}
\hline $\begin{array}{l}\text { Bravais: } \\
\{0001\}\end{array}$ & $\begin{array}{l}\text { Miller: } \\
\{1 \mid 1\}\end{array}$ & $\begin{array}{c}\text { Na umann: } \\
0 h^{3}\end{array}$ & $\begin{array}{c}\text { Lé v : : } \\
a^{1}\end{array}$ & $\begin{array}{c}\text { Buchstabc: } \\
e\end{array}$ \\
\hline$\{10 \overline{1} 0\}$ & $\{2 \bar{T}\}$ & $\infty l i$ & $e^{2}$ & $m$ \\
\hline$\{1011\}$ & $\{100\}$ & $I$ & $p$ & $r$ \\
\hline$\{0111\}$ & $\{22 T\}$ & $-I i$ & $e^{\frac{1}{2}}$ & $\approx$ \\
\hline$\{20 \overline{2} 1\}$ & $\{011\}$ & $2 R$ & $e^{5}$ & $\sigma$ \\
\hline$\{02 \bar{\Phi} 1\}$ & $\{11 \overline{1}\}$ & $--2 R$ & $e^{1}$ & $s$ \\
\hline$\{30 \overline{3} 4\}$ & $\{10.1 .1\}$ & $3 R$ & $a^{10}$ & $t$ \\
\hline$\{03 \overline{3} 4\}$ & $\{77 \bar{\Phi}\}$ & $-3 R$ & $e^{\frac{2}{7}}$ & $\tau$ \\
\hline
\end{tabular}

Meine Krystalle bestehen oft aus mehreren Indjviducn in paralleler Jortwachsung. Bald sind die Axen $\theta$ der vorschiedenen Krystalle genau parallel, bald sind sie etwas unter einander gencigt. Ungenaue parallele Fortwachsungen sind in der Natur viel verbreiteler als man glaubt. Vor Kurzem hat I. Brugnatelli') solche annähernd parallele fortwachsungen am Brookit von Piattagrande gefunden, und ich selbst ${ }^{2}$ ) habe das an einem Epidotkrystalle vom Sulzbachthale beschrieben. Nach meinen eigenen, nicht veröffentlichten Untersuchungen ist dieses Phänomen am schwarzen $\Lambda$ ugit der Allaner Gebirge und des Vesuvs (Aushruch von 1872) und am Manganit von Ilefeld ziemlich häulig.

Die Basis ist fast immer sehr regelmässig, alser etwas malt, die Prismenflächen sind oft uneben und krumm. Unter der Biegung hat der Krystall bald ohne zu springen sich gekrümmt, bald in den Maximis der Krümmung Risse bckommen, wie es an den Quarzkrystallen von Pitourlesen-I ordat (Arìge) der Fall ist, welche $\Lambda$. Lacroix ${ }^{3}$ ) beschrieb.

Meine chemischen Untersuchungen haben folgende Resultate gegebe n

1) Ottaedrite e brookite della Pialtagrande, presso Sondalo in Valtellina. lendiconti R. Istit. Lomb. di lett. scienze ed arti 1900, 32, 1045. Auch diese Zeitschr. $1900,32,353$.

2) Leber zwei llächenceiche Epidolkrystalle von Sulzbach. N. Jahrb. I. Min., Geol. u. s. w. $1900,1,181$.

3) Sur les deformations subies par les cristaux de quartz des filons de pilourles-en-Lordat elc. Bull. de la soc. franç. de min. 1894, 14, 306. Ausz. diese Zeitschr. 22, 58 s. 


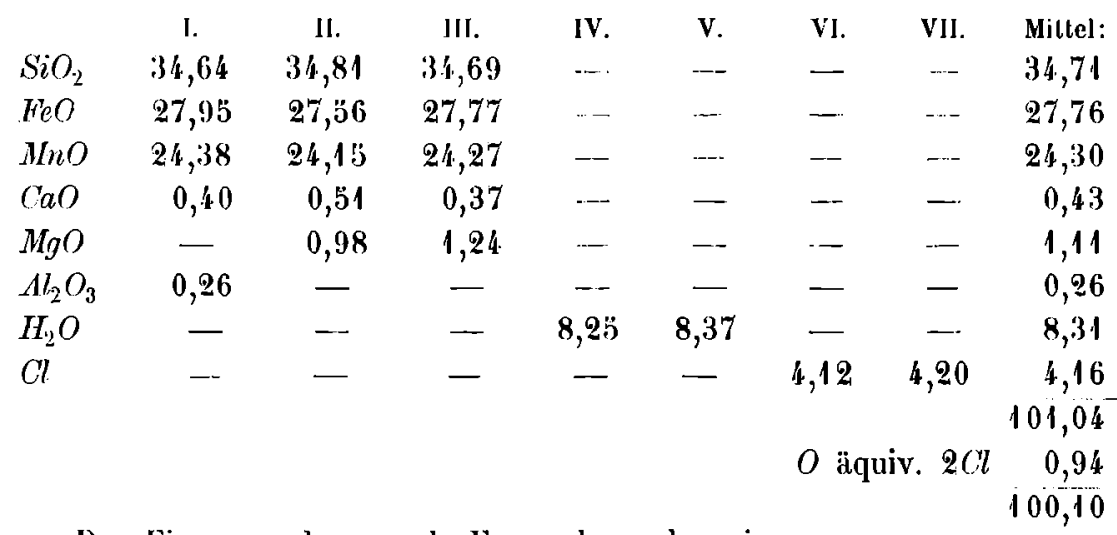

Das Eisen wurde nur als Ferrosalz nachgewiesen.

Wenn wir alle zweiwerthigen Elemente in $\mathrm{FeO}$ vereinigen und die kleine Menge Thonerde vernachlässigen, so wird meine Analyse:

$\begin{array}{lrlc}\mathrm{SiO} \mathrm{O}_{2} & 34,10 & 0,568 & 9,9 \\ \mathrm{HeO} & 50,42 & 0,700 & 12,2 \\ \mathrm{Hi}, \mathrm{O} & 8,17 & 0,454 & 7,9 \\ \mathrm{Fe} & 3,22 & 0,0575 & 1 \\ \mathrm{Cl} & 4,09 & 0,115 & 3\end{array}$

Die diesen Zahlen enlsprechende Formel ist also

$$
\mathrm{RCl}_{2} .12 \mathrm{RO} .10 \mathrm{SiO}_{2}+8 \mathrm{H}_{2} \mathrm{O} \text {, }
$$

welche erfordert (wenn $\mathrm{RO}=\mathrm{Fe}$ ) :

\begin{tabular}{lrc} 
& & $\delta$ \\
$\mathrm{SiO}_{2}$ & 34,58 & 0,48 \\
$\mathrm{FeO}_{C}$ & 49,80 & 0,62 \\
$\mathrm{H}_{2} \mathrm{O}$ & $\mathbf{8 , 3 0}$ & 0,13 \\
$\mathrm{He}$ & 3,23 & 0,04 \\
$\mathrm{Cl}$ & $-4,09$ & - \\
\cline { 2 - 2 } & $-100,00$ & 1,24
\end{tabular}

Diese Formel ist in befriedigender Uebereinstimmung auch mit den anderen, vorhin erwähnten Analysen, und sie kann daher als die genaue Formel des Pyrosmalith betrachtet werden.

Auch die chemische Zusammensetzung des mit dem Pyrosmalith isomorphen Friedelit ist durch diese Formel darstellbar. Von diesem letzteren besitzen wir bis jetzt vier genaue Analysen. A. Gorgeu ${ }^{1}$ ) hat die Proben von Adervielle analysirt, F. Flink ${ }^{2}$ ) und (G. Lindström ${ }^{3}$ ) jene von 1) a. a. 0 .

2) Mineralogiska Notiser. Bihang till k. Svenska Vet.-Akad. Handlingar 1890, 16, Afd. II, No. 43, 1. Ausz. diese Zeitschr. 20, 374.

3) Mineralanalyser. Geol. Foören. i Stockholm Förh. 1891, 13, 123. Ref. diese Zeitschr. 23, 155 . 
Harstigen, L. J. Igelström¹) die Varietät der Sjögrube. Diese Gelehrten fanden:

\begin{tabular}{|c|c|c|c|c|}
\hline $\mathrm{SiO}_{2}$ & $\begin{array}{c}\text { G orge u: } \\
34,45\end{array}$ & $\begin{array}{l}\text { Flink: } \\
34,66\end{array}$ & $\begin{array}{l}\text { Lindströ in: } \\
\quad 33,36\end{array}$ & $\begin{array}{c}\text { Igelström } \\
\mathbf{3 4 , 3 6}\end{array}$ \\
\hline $\mathrm{FeO}$ & Spur & 4,08 & 3,83 & 1,35 \\
\hline $\mathrm{MnO}$ & 51,99 & 46,52 & 49,08 & 49,48 \\
\hline $\mathrm{CaO}$ & 0,40 & 0,53 & 0,74 & 1,50 \\
\hline $\mathrm{MgO}$ & 1,20 & 2,27 & 1,31 & 1,50 \\
\hline $\mathrm{Cl}$ & 3,10 & 4,04 & 4,19 & 3,00 \\
\hline $\mathrm{H}_{2} \mathrm{O}$ & 9,60 & 8,47 & 8,45 & 9,00 \\
\hline \multirow[t]{2}{*}{$P_{2} O_{8}$} & - & - & Spur & - \\
\hline & $100,74^{\circ}$ & 100,57 & 100,96 & 100,19 \\
\hline \multicolumn{2}{|c|}{ äquiv. $2 C l=0,70$} & 0,92 & 0,95 & 0,68 \\
\hline & 100,04 & 99,65 & 100,01 & 99,91 \\
\hline
\end{tabular}

Diese Analysen zeigen eine völlige Uebereinstimmung mit der Formel $\mathrm{RCl}_{2} .12 \mathrm{RO} .10 \mathrm{SiO}_{2}+8 \mathrm{H}_{2} \mathrm{O}$,

welche also die allgemeine Formel des Friedelit und Pyrosmalith ist.

\section{Apophyllit von der Seiser-Alpe.}

(Hierzu Tafel VIII, Fig. 11.)

1891 hat P. J. Ploner ${ }^{2}$ ) die Krystalle dieses Fundortes (Cipit- und Frombach) sorgfältig beschrieben; seine Arbeit ist auch wichtig für die grosse Zahl bibliographischer Nachrichten über die früheren Untersuchungen von Lévy ${ }^{3}$ ), Richthofen ${ }^{4}$ ), Des Cloizeaux ${ }^{5}$ ), Schrauf ${ }^{6}$ ), Rumpf ${ }^{7}$ ) u. s. w.

Er hat zahlreiche neue Formen beobachtet, welche häufig einem complicirten Symbol entsprechen. Ich werde hier nur einen Krystall beschreiben, welcher mir einige seltene Formen bot.

Er ist stark tafelfürmig nach der Basis $\{001\}$ und zeigt die gewöhnlichen Formen $\{001\},\{100\},\{111\}$, ferner sind die Combinationskanten von

1) Friedelit aus der Sjögrube (Hausmannit-, Braunit- und Eisenerzgrube), Grythytte, Kirchspiel Örebro. Diese Zeitschr. 1893, 21, 92. 18, 337 .

2) Ueber die Krystallformen des Apophyllits der Seiseralpe. Diese Zeitschr. 1891,

a) Déscription d'une collection de minéraux formée par M. Henri Heuland. London 1837, 2, 271.

4) Ueber Bildung und Umbildung einiger Mineralien in Südlirol. Sitzungsber. d. k. Akad. Wien $1857,27,359$.

5) Manuel de minéralogie, Paris $1862,1$.

6) Apophyllitzwillinge von Grönland. Sitz.-Ber. d. k. Akad. Wien 1870, 62, 700.

7) Ueber den Krystallbau des $\Lambda$ pophyllits. Tschermak's min. und petrogr. Mittheilungen 1880, 2, 369. Ref. diese Zeitschr. 5, 374. 
(010) und \{001\} durch zwei genügend grosse Flächen abgestumpft, welche den Symbolen (013) und (015) entsprechen.

Die Messungen gaben:

$$
\begin{aligned}
& (010):(013)=67^{1} 16^{\prime} \\
& (010):(01 \overline{3})=6722
\end{aligned}
$$

Aus den Miller'schen Constanten

folgt:

$$
a: a: c=1: 1: 1,2515
$$

$$
(010):(013)=67^{\circ} 21^{\prime} \text {. }
$$

Die Deuteropyramide $\{103\}$ wurde von Ploner entdeckt.

Es ist nun sehr wichtig, dass die Combinationskanten von (100) und \{001\} anders als jene von (010) mit der Basis abgestumpft sind. In der That, während die Kante $[100: 00 T]$ keine Fläche bietet, finden sich an der Kante [100:001] zwei Linearflächen, welche genaue Messungen lieferten.

Die eine ist (106), welche Rumpf entdeckt und Ploner an vielen Krystallen gefunden hat; die andere ist eine zu $\{103\}$ vicinale Fläche, welcher das complicirte und am Apophyllit unbekannte Symbol (7.0.20) zukommt.

$$
\begin{aligned}
& (100):(106)=78^{021} 1^{\prime} \text { gem. } 78^{\circ} 13^{\prime} \text { ber. } \\
& (100):(7.0 .20)=6626-6621 \text { - }
\end{aligned}
$$

Die Basis dieses Krystalles besitzt einen sehr complicirten Bau, wie die verschiedenen Streifen ihrer Oberfläche zeigen. Fig. 11 Taf. VIII giebt ein genaues Bild der Fläche (001). 